Original Article

\title{
Intra- and inter-examiner reliability of goniometer and inclinometer use in Craig's test
}

\author{
Bo-RAm ChOI, PhD, $\mathrm{PT}^{1)}$, Sun-Young Kang, MSc, $\left.\mathrm{PT}^{2}\right)^{*}$ \\ 1) Department of Physical Therapy, College of Medical and Life Science, Silla University, Republic of \\ Korea \\ 2) Department of Physical Therapy, Graduate School, Yonsei University: 234 Maeji-ri, \\ Heungeop-myeon, Wonju-si, Gangwon-do, Republic of Korea
}

\begin{abstract}
Purpose] The purpose of this study was to determine the inter- and intra-examiner reliability of measurement methods for femoral anterversion during Craig's test. [Subjects and Methods] The study included 37 healthy participants (20 males and 17 females). Two novice examiners (Department of Physical Therapy students at Silla University) used three different methods to measure the femoral anterversion during Craig's test: a goniometer, a goniometer with a laser beam, and an inclinometer. [Results] The intra-examiner reliability was high for both examiners with all three measurement methods, with scores of $0.82,0.86$, and 0.73 for examiner 1 and $0.74,0.78$, and 0.72 for examiner 2 for the goniometer, goniometer with the laser beam, and inclinometer, respectively. The inter-examiner reliability during Craig's test was below moderate for both the goniometer $(0.25)$ and inclinometer (0.27) and moderate for the goniometer with the laser beam (0.62). [Conclusion] This study found that Craig's test using a goniometer with a laser beam had high intra-examiner reliability and moderate inter-examiner reliability. Clinically, these findings may supplement existing measurement skills and reduce the difficulty of locating the goniometer axis during Craig's test.
\end{abstract}

Key words: Craig's test, Goniometer with a laser, Inclinometer

(This article was submitted Oct. 24, 2014, and was accepted Dec. 11, 2014)

\section{INTRODUCTION}

Lower extremity (LE) malalignment influences the load distribution on the joints, mechanical efficiency of the muscles, and proprioceptive orientation and feedback from the hip and knee, resulting in altered neuromuscular function and control of the lower extremities ${ }^{1,2)}$. Among these LE alignment variables, femoral anteversion has been identified as a risk factor for hip and knee joint injury ${ }^{3,4)}$. Increased femoral anteversion can increase hip adduction and knee abduction because the patella shifts to the medial side of the femoral condyle groove, thereby increasing the Q-angle and ultimately resulting in knee valgus deformity ${ }^{5}$. As females have a wider pelvis, larger Q-angle, and larger femoral anteversion values than males, more females experience frontal plane stress while engaging in functional tasks ${ }^{4}$. Thus, an accurate assessment of femoral anteversion is important for diagnosing and preventing hip and knee injuries ${ }^{6}$.

Femoral anteversion is the degree of forward projection of the femoral neck from the frontal plane of the shaft ${ }^{7}$. Various methods and equipment have been used to measure

\footnotetext{
*Corresponding author. Sun-young Kang (E-mail: monocromtoy@hotmail.com)
}

C2015 The Society of Physical Therapy Science. Published by IPEC Inc. This is an open-access article distributed under the terms of the Creative Commons Attribution Non-Commercial No Derivatives (by-ncnd) License $<$ http://creativecommons.org/licenses/by-nc-nd/3.0/> . femoral anteversion. Radiographic evidence and complex trigonometric formulae have been employed to determine the femoral anteversion angle and the femoral neck shaft angle ${ }^{8)}$. However, the limitations of radiographs do not allow for the axes to be placed accurately ${ }^{9}$. Ultrasound (US), which is both inexpensive and noninvasive, can be used to measure femoral anteversion, but it is not easy for a novice examiner to find the true axis of the femur ${ }^{10)}$. Computed tomography (CT) is one of the most reliable methods of measurements for determining the rotational alignment of the lower extremities ${ }^{11}$. The inter-examiner reliability of femoral anteversion measurements with $\mathrm{CT}$ is higher than with magnetic resonance imaging (MRI), and there is a significant correlation between the results of CT and the internal rotation of the hip joint. Although imaging can be useful, a physical examination is needed in daily clinical practice to determine femoral anteversion. Craig's test is the most commonly used physical method for measuring femoral anteversion. Craig's test results correlate with commonly used radiographic techniques $\left.(r=0.93)^{12}\right)$. Studies have reported that Craig's test has high intra-examiner reliability. However, the examiner may find it difficult to locate and palpate the greater trochanter and the goniometer axis because one hand has to maintain the internal rotation of the hip while the other hand measures the angle between a virtual vertical line, which is based on different standards among examiners and the bisection line of the tibia using the goniometer. Examiners may find it difficult to control the goniometer axis while maintaining the maximal lateral 
Table 1. $\mathrm{ICC}_{3,1}$ and $95 \% \mathrm{CI}$ for day-to-day intra-examiner reliability

\begin{tabular}{lccccc}
\hline \multirow{2}{*}{ Session } & \multicolumn{2}{c}{ Examiner 1 } & \multicolumn{2}{c}{ Examiner 2 } \\
\cline { 3 - 6 } & & \multicolumn{1}{c}{ Mean (SD) } & ICC (95\%CI) & Mean (SD) & ICC (95\%CI) \\
\hline \multirow{2}{*}{ Goniometer } & 1 & $10.5 \pm 1.4^{*}$ & $0.82(0.61$ to 0.92$)$ & $7.9 \pm 1.2$ & $0.74(0.43$ to 0.88$)$ \\
\multirow{2}{*}{ Goniometer with a laser beam } & 2 & $10.4 \pm 1.2$ & & & \\
& 1 & $10.3 \pm 1.0$ & $0.86(0.68$ to 0.93$)$ & $10.0 \pm 1.2$ & $0.78(0.51$ to 0.9$)$ \\
Inclinometer & 2 & $10.0 \pm 1.2$ & & $9.7 \pm 1.3$ & \\
& 1 & $9.6 \pm 1.3$ & $0.73(0.39$ to 0.88$)$ & $8.7 \pm 1.9$ & $0.72(0.37$ to 0.87$)$ \\
\hline
\end{tabular}

*Degree

position of the greater trochanter.

Thus, there is a continuing need for a method to measure femoral anteversion that is easy to use, accurate, and reproducible. The purpose of this study was to examine the inter- and intra-examiner reliability of a goniometer, an inclinometer, and a goniometer with a laser, all of which are tools used to measure femoral anteversion in Craig's test.

\section{SUBJECTS AND METHODS}

Thirty-seven healthy volunteers (20 males, age $28.7 \pm 1.1$ years, height $1.71 \pm 0.4 \mathrm{~m}$, and mass $72.8 \pm 7.7 \mathrm{~kg}$; 17 females, age $26.3 \pm 1.6$ years, height $1.64 \pm 0.2 \mathrm{~m}$, and mass $67.3 \pm 5.9 \mathrm{~kg}$ ) participated. The inclusion criteria were no prior medical history of surgery and no acute LE orthopedic injury or lumbar spine injury for at least the previous 6 months. Before taking part, the participants provided written informed consent. This study was approved by the Institutional Review Board at Silla University.

We used three methods to measure the femoral anteversion. The first method employed the traditional Craig's test. The participants were placed in a prone position, with $90^{\circ}$ flexion of the knee joint. Using one hand, the examiner palpated the greater trochanter until it was positioned laterally and maximally during passive internal rotation of the hip joint. The examiner then used a handheld goniometer with his other hand to measure the angle between the tibial lines, a line bisecting the medial and lateral malleoli, and an imaginary vertical line extending from the table ${ }^{13,14}$ ).

The second method also started with Craig's test but used an Acumar digital inclinometer (Lafayette Instrument Co., Lafayette, IN, USA) instead of a handheld goniometer. The inclinometer was attached along the line between the fibular head and the lateral malleolus. Calibration was performed with the vertical line extending from the table during each Craig's test, and the angle displacement between the vertical line and the end position was recorded.

In the third method, femoral anteversion was measured using a handheld goniometer with a leveling laser beam (LD-SL01, LAND Group, Zhejiang, PR China). The leveling laser beam, located on a tripod, was used to project both vertical and horizontal beams. The intersection point of the vertical and horizontal lines was focused on the center of the subject's patella. During Craig's test, the position of the goniometer axis for the virtual vertical line was located using the vertical laser beam.
Two novice examiners (fourth-year undergraduates in the Department of Physical Therapy) used the three methods in all the tests. The measurement methods were standardized for both examiners in a pilot test. The examiners' results from the first session were compared with the corresponding results from the second session to calculate the intraexaminer reliability of each method. To avoid any learning effect, each examiner had a 1-day interval between the first and second sessions. On the same day, the other examiner evaluated the same subject in an identical fashion to calculate the inter-examiner reliability. All the data were recorded by a third examiner to avoid comparison of the data during the three methods for each of the two examiners. The examiners collected the data during the tests to ensure that the participants remained as stable as possible for all three methods. To ensure that the examiners remained blinded to each other's assessments, the two examiners entered the examination room independently. Each of the examiners performed three trials (one session for each trial), and each measurement was taken on the right femur. The order of the methods was randomized.

The data were analyzed using PASW Statistics for Windows (Version 18.0; SPSS Inc., Chicago, IL, USA). Intraclass correlation coefficients (ICC) $(3,1)$ and 95\% confidence intervals $(\mathrm{CI})$ were used to analyze the intraexaminer and inter-examiner reliability. The ICC reference values of the present study for the reliability of the measurements were poor (less than 0.25$)$, low (0.26-0.49), moderate $(0.50-0.69)$, high $(0.70-0.89)$, and very high (above 0.90$)^{15)}$.

\section{RESULTS}

The reliability of both examiners was high with all the Craig's test methods. The goniometer, combined with the laser, showed the highest reliability (ICC 0.68-0.93) among the methods assessed (Table 1). The reliabilities of both the goniometer and the inclinometer were low. However, the Craig's test with the goniometer and laser showed moderate reliability (ICC 0.13-0.83) (Table 2).

\section{DISCUSSION}

The aim of this study was to determine the intra- and inter-examiner reliabilities of femoral anteversion measurements obtained using Craig's test with a goniometer, an inclinometer, and a goniometer with a laser beam. The 
Table 2. $\mathrm{ICC}_{3,1}$ and $95 \% \mathrm{CI}$ for inter-examiner reliability of day 1

\begin{tabular}{ll}
\hline & \multicolumn{1}{c}{ ICC $(95 \% \mathrm{CI})$} \\
\hline Goniometer & $0.25(-0.68$ to 0.67$)$ \\
Goniometer with a laser beam & $0.62(0.13$ to 0.83$)$ \\
Inclinometer & $0.27(-0.64$ to 0.68$)$ \\
\hline
\end{tabular}

results showed that Craig's test using a goniometer with a laser beam had moderate inter-examiner reliability and that the reliability was higher than that of the other methods.

Various techniques have been used to measure femoral anteversion. Ryder's method ${ }^{8)}$ is similar to Craig's test in terms of the goniometer axis. The angle between a line extending from the longitudinal axis of the tibia, assumed to be vertical, to the dorsal condyle of the femur and a vertical line to the ground is considered to represent femoral anteversion. The femoral torsion index (FTI) is another femoral anteversion measure. It is considered easier to find the femoral torsion. Rather than finding the longitudinal axis of the tibia, the femoral torsion axis is found using a line connecting the dorsal surfaces of the medial and lateral condyle of the femur. Although the FTI is more reliable than Ryder's method ${ }^{16)}$, we focused on the reliability of the vertical line extending to the ground. Although both Ryder's method and the FTI utilize the longitudinal axis of the femur, the imaginary vertical line could vary with the standing posture and head posture of the rater. The results of our study showed that Craig's test using a goniometer with a laser beam was more reliable than the traditional Craig's test. The laser provides a reference axis for the imaginary vertical line.

Craig's test has shown high reliability in measuring femoral anteversion ${ }^{12,15)}$. Ruwe et al. reported that Craig's test of the femoral anteversion angle correlated with intraoperative measures $(r=0.930)$ and with radiographic techniques $(r$ $=0.941$ ). However, other studies found that the results of physical examinations of femoral anteversion were not consistent with those obtained with imaging techniques ${ }^{10,16)}$. Souza and Powers ${ }^{17)}$ reported that physical examinations had such a wide confidence interval that their clinical utility may even be called into question. One problem when using a goniometer is the position of the axis. Although goniometers are commonly used to measure the range of motion, it is difficult to simultaneously handle the instrument and identify reference anatomical points. Additionally, anatomical errors and undesirable movements may compromise the results $^{18,19)}$. Another possible reason why goniometers are less reliable is that the experience and ability of the examiner may, at least partially, affect the reproducibility of the measurement ${ }^{20}$. Our results showed that the inclinometer method with Craig's test had lower reliability because of the difficulty in locating the attachment position for the inclinometer. We used a goniometer with a laser beam to increase the reproducibility in assessing the vertical axis, resulting in better inter-examiner reliability than the traditional Craig's test. Using a goniometer with a laser beam, the novice examiner can measure femoral anteversion with a more accurate reference axis. However, the examiner cannot ensure the femoral anteversion angle using a goniometer with a laser beam because physical examination methods do not correlate well with imaging techniques (CT, MRI, US) ${ }^{10,16)}$. Clinicians are aware that goniometer measurements are not interchangeable with true femoral torsion obtained by imaging techniques.

Although we controlled the standard measurement axis with the laser beam, we did not control subject posture during the measurement. Pelvic location was not in the same prone position on the two measurement days. Because the acetabulum location is changed by the pelvic tilt position ${ }^{21)}$, the pelvic location could affect the range of motion in femur rotation. Additionally, no lower extremity muscle length test was performed; thus, the iliopsoas muscles and rectus femoris can affect the pelvic and femur locations in a prone position. The future studies should consider participants' positions during measurement and examine relationship between muscle length in lower extremity and femoral anteversion.

In this study, we found that Craig's test using a goniometer with the laser beam method showed high intra-examiner reliability and moderate inter-examiner reliability. Clinically, these findings may supplement existing measurement skills and reduce the difficulty in locating the goniometer axis during Craig's test.

\section{REFERENCES}

1) Sharma L, Song J, Felson DT, et al.: The role of knee alignment in disease progression and functional decline in knee osteoarthritis. JAMA, 2001, 286: 188-195. [Medline] [CrossRef]

2) Shultz SJ, Nguyen AD, Levine BJ: The relationship between lower extremity alignment characteristics and anterior knee joint laxity. Sports Health, 2009, 1: 54-60. [Medline] [CrossRef]

3) Tönnis $D$, Heinecke A: Acetabular and femoral anteversion: relationship with osteoarthritis of the hip. J Bone Joint Surg Am, 1999, 81: 1747-1770. [Medline]

4) Medina McKeon JM, Denegar CR, Hertel J: Sex differences and discriminative value of lower extremity alignments and kinematics during two functional tasks. J Appl Biomech, 2010, 26: 295-304. [Medline]

5) McLean SG, Huang X, van den Bogert AJ: Association between lower extremity posture at contact and peak knee valgus moment during sidestepping: implications for ACL injury. Clin Biomech (Bristol, Avon), 2005, 20: 863-870. [Medline] [CrossRef]

6) Kwon O, Yun M, Lee W: Correlation between intrinsic patellofemoral pain syndrome in young adults and lower extremity biomechanics. J Phys Ther Sci, 2014, 26: 961-964. [Medline] [CrossRef]

7) Magee DJ: Orthopedic physical assessment, 5th ed. Philadelphia: Saunders Co., 2009.

8) Ryder CT, Crane L: Measuring femoral anteversion; the problem and a method. J Bone Joint Surg Am, 1953, 35-A: 321-328. [Medline]

9) Parikh S, Noyes FR: Patellofemoral disorders: role of computed tomography and magnetic resonance imaging in defining abnormal rotational lower limb alignment. Sports Health, 2011, 3: 158-169. [Medline] [CrossRef]

10) Botser IB, Ozoude GC, Martin DE, et al.: Femoral anteversion in the hip: comparison of measurement by computed tomography, magnetic resonance imaging, and physical examination. Arthroscopy, 2012, 28: 619627. [Medline] [CrossRef]

11) Tomczak RJ, Guenther KP, Rieber A, et al.: MR imaging measurement of the femoral antetorsional angle as a new technique: comparison with CT in children and adults. AJR Am J Roentgenol, 1997, 168: 791-794. [Medline] [CrossRef]

12) Ruwe PA, Gage JR, Ozonoff MB, et al.: Clinical determination of femoral anteversion. A comparison with established techniques. J Bone Joint Surg Am, 1992, 74: 820-830. [Medline]

13) Daneshmandi H, Saki F, Shahheidari S, et al.: Lower extremity Malalignment and its linear relation with Q angle in female athletes. Procedia Soc Behav Sci, 2011, 15: 3349-3354. [CrossRef]

14) Kaneko M, Sakuraba K: Association between femoral anteversion and lower extremity posture upon single-leg landing: implications for anterior 
cruciate ligament injury. J Phys Ther Sci, 2013, 25: 1213-1217. [Medline] [CrossRef]

15) Jonson SR, Gross MT: Intraexaminer reliability, interexaminer reliability, and mean values for nine lower extremity skeletal measures in healthy naval midshipmen. J Orthop Sports Phys Ther, 1997, 25: 253-263. [Medline] [CrossRef]

16) Tamari K, Tinley P, Briffa K, et al.: Validity and reliability of existing and modified clinical methods of measuring femoral and tibiofibular torsion in healthy subjects: use of different reference axes may improve reliability. Clin Anat, 2005, 18: 46-55. [Medline] [CrossRef]

17) Souza RB, Powers CM: Concurrent criterion-related validity and reliability of a clinical test to measure femoral anteversion. J Orthop Sports Phys Ther, 2009, 39: 586-592. [Medline] [CrossRef]
18) Szulc P, Lewandowski J, Marecki B: Verification of selected anatomic landmarks used as reference points for universal goniometer positioning during knee joint mobility range measurements. Med Sci Monit, 2001, 7 : 312-315. [Medline]

19) Gerhardt JJ, Rondinelli RD: Goniometric techniques for range-of-motion assessment. Phys Med Rehabil Clin N Am, 2001, 12: 507-527. [Medline]

20) dos Santos CM, Ferreira G, Malacco PL, et al.: Intra and inter examiner reliability and measurement error of goniometer and digital inclinometer use. Rev Bras Med Esporte, 2012, 18: 38-41.

21) Kalteis TA, Handel M, Herbst B, et al.: In vitro investigation of the influence of pelvic tilt on acetabular cup alignment. J Arthroplasty, 2009, 24 152-157. [Medline] [CrossRef] 